\title{
Betel-Zeremonie und -Bräuche in der Minang-Kultur Indonesiens
}

\author{
Herwig ZahorKa
}

\begin{abstract}
Betel bites are widespread in Asia. They contain seeds of betel palm and some other ingredients. The preparation of a traditional betel bite from Sumatra is described. For the production very specific tools as well as special betel scissors are used.
\end{abstract}

\section{Zusammenfassung}

In Asien weit verbreitet ist der Betelbissen. Er enthält Samen der Betelpalme und einige andere Zutaten. Beschrieben wird die Zubereitung eines traditionellen Betelbissens aus Sumatra. Für die Herstellung werden ganz bestimmte Werkzeuge wie auch spezielle Betelscheren verwendet.

\section{Der Betelbissen - ein anregendes Genussmittel}

Wurde ein Gast in einem Hause des mutterrechtlichen Stammes der Minangkabau in West-Sumatra empfangen, so bot ihm die Frau des Hauses ein Gefäß mit frischen Sirih-Blättern an. Gekaut, empfindet er einen aromatischen erfrischenden Geschmack. Aber das ist nicht alles. Es werden noch mehr Zutaten angeboten. Der Gastgeber bietet sie an und macht selbst Gebrauch davon.

Er nimmt zwei oder drei Sirih-Blätter, legt in die Mitte das Viertel einer Betelnuss und dazu etwas Gambir (auch Gambier geschrieben) und streut eine dicke Prise gemörserten Kalk darüber.

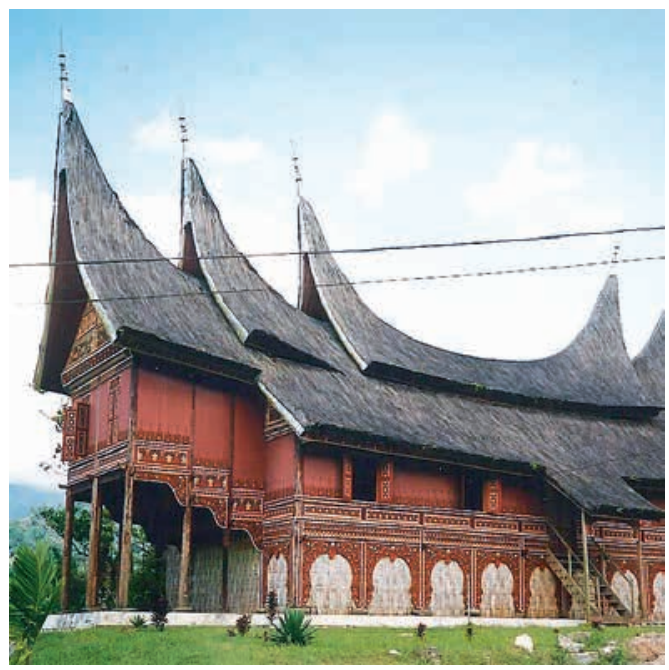

Abb. 1: Traditionelles Minang-Haus vom Piliang Typ.

(Foto: H. ZAHORKA)
Meist kommt noch eine Portion Tabak dazu. Er faltet die Blätter über den Zutaten zu einem Päckchen - und der Betelbissen ist fertig. Er wird in den Mundwinkel hinter die Backe geschoben und manchmal auch gekaut. Seltenere aromatische $\mathrm{Zu}$ taten sind Anis, Gewürznelken, Kardamom, Zimt oder Minze.

Es entwickelt sich ein vermehrter rotgefärbter Speichelfluss mit herb-aromatischem und leicht adstringierendem Effekt, der Hunger und Durst unterdrückt und leicht euphorisch wirkt, weil das zentrale Nervensystem stimuliert wird. Bei langzeitigem Gebrauch färben sich die Zähne rot und später schwarz.

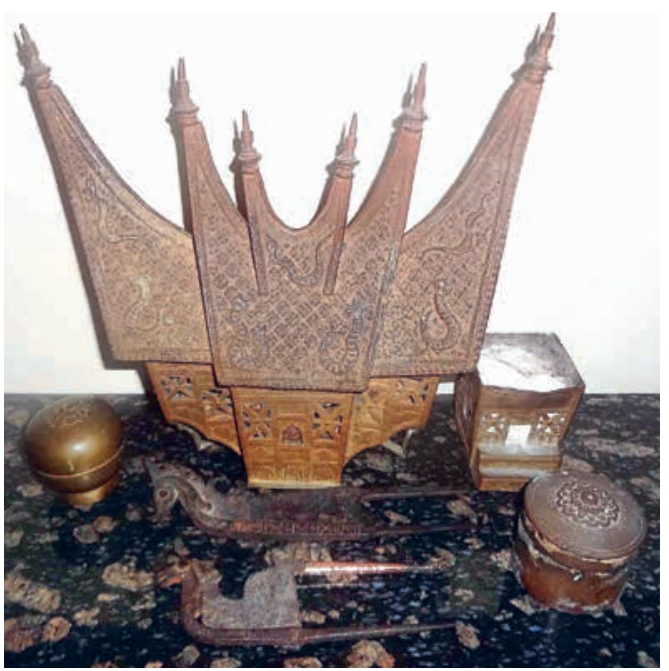

Abb. 2: Minang Sirih Pinang-Ensemble mit Betelscheren aus dem 19. Jahrhundert. (Foto: H. ZaHorka) 


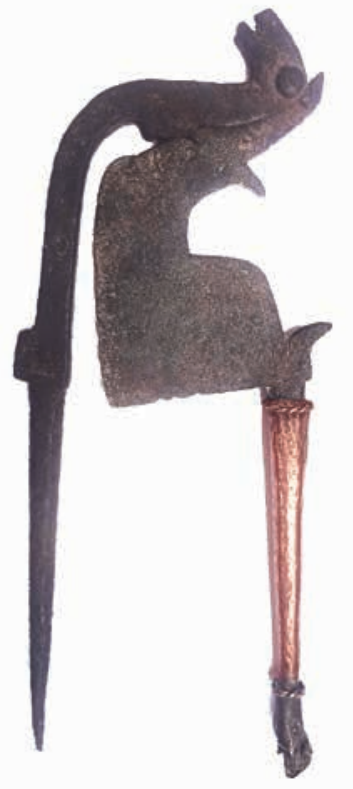

Abb. 3: Minang Betelschere. (Foto: H. Zahorka)

Dieser Brauch ist in S- und SO-Asien weit verbreitet, von Indien über den Melanesischen Archipel bis zu den Pazifikinseln, und er ist in diesen tropischen und subtropischen Regionen seit der Jungsteinzeit nachweisbar. Dieses Genussmittel wird auch bei Feierlichkeiten wie Hochzeiten, Geburtsfeiern und Bestattungsritualen angeboten. Die Minangs sagten: Es öffnet die Tür des Gastgebers und die Herzen der Gäste.

In den urbanen Gebieten haben die Zigaretten den Sirih-Betel Priem längst vertrieben. In Indonesien ist er aber noch ein tägliches Stimulans auf den Außeninseln wie Nias, Sumba, Flores, Timor oder Alor.

\section{Das Ritualgeschirr}

Ein rituell dem Gast angebotenes Genussmittel erfordert auch ein repräsentatives Ritualgeschirr. Es besteht meistens aus einem rechteckigen Kästchen für die Sirih-Blätter und aus kleineren runden Gefäßen für Kalk und Gambir und gegebenenfalls für Tabak. All diese Behälter sind mit einem Deckel versehen. Die Betelnüsse liegen meist auf einem Tablett. Das Material dieses Geschirrs ist in der Regel Messing oder Bronze, bei wohlhabenden Familien aber auch Silber. Zum Ensemble gehören

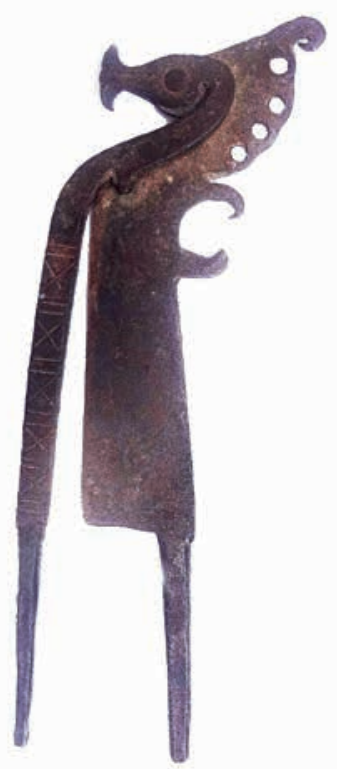

Abb. 4: Eine weitere Minang Betelschere aus dem 19. Jahrhundert. (Foto: H. ZAHORKA)

Betelscheren zum Vierteilen der Betelnüsse. Der Kopf dieser Schneidwerkzeuge hat oft künstlerische Formen, zum Beispiel die eines Steckenpferdes. Dazu kommt noch ein kleiner Mörser zum Pulverisieren des Kalkes.

Die indonesische Ehefrau des Autors hat von ihrer Minang-Urgroßmutter ein besonderes Ensemble für den Betel-Kult aus dem 19. Jahrhundert geerbt. Es besteht aus einem traditionellem Miniatur-Minanghaus vom Piliang-Typ (Herrenhaus),

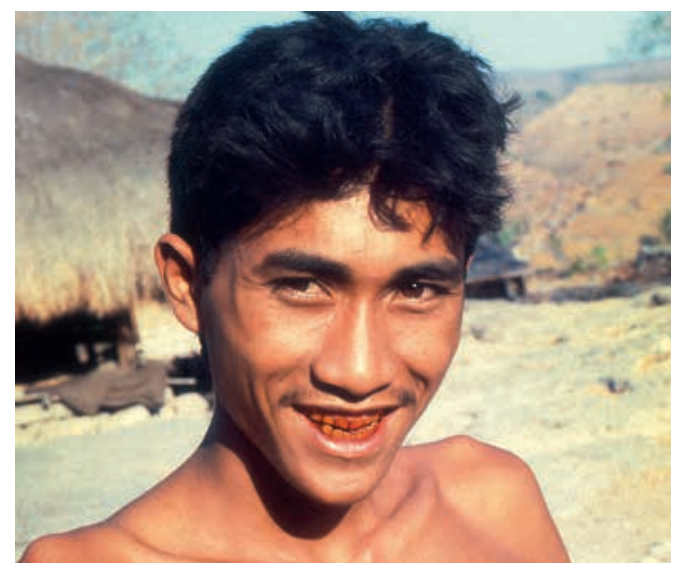

Abb. 5: Betelkauen verfärbt Zähne und Zahnfleisch rot. (Foto: H. ZaHORKA) 


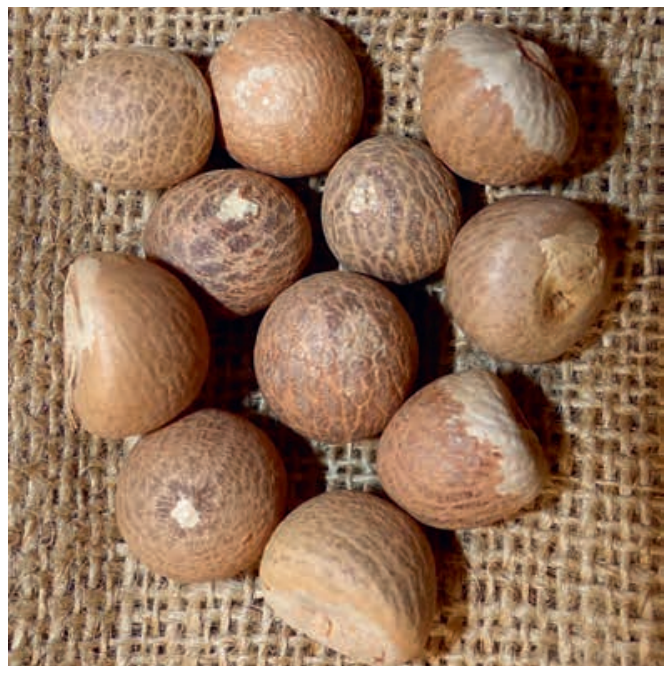

Abb. 6: Handelsübliche unzerkleinerte Betelnüsse aus Indien. (Foto: H. Steinecke)

$25 \mathrm{~cm}$ breit, und drei kleineren Gefäßen. Das Material ist wahrscheinlich Bronze. Der mittlere Teil des Daches ist abnehmbar. Im Inneren des Hauses befinden sich noch uralte eingetrocknete Betelnüsse, trockene Sirih-Blätter, etwas Gambir und in Stanniol eingewickelter Tabak. Die beiden kleinen runden Behälter sind wahrscheinlich für Gambir und Tabak vorgesehen. Dem kantigen Miniatur-Reisspeicher fehlt das Dach. Er ist noch immer mit eingepacktem Kalkpulver gefüllt.
Das Auffallende am Dach ist das Design der Oberfläche. Hier sind mehrere Nagas dargestellt, die fast die Form von Würmern haben. Nagas sind mythische hinduistische Schutz-Gottheiten in Schlangenform, die hier ihre schützende und fruchtbarkeitsverheißende Kraft für die Hausbewohner entfalten sollen. Auffallend ist dies deshalb, weil die Minangs bereits Anfang des 19. Jahrhunderts von Imam Bonjol vom Hinduismus zum Islam konvertiert wurden, nicht immer mit friedlichen Mitteln.

\section{Literatur}

Bayer, C. \& Steinecke, H. 2018 (Red.): Palmen. - Palmengarten-Sonderheft 50. - Frankfurt

Lauer, H. H. 1966: Zur Tradition exotischer Drogen: Faufal (Areca catechu L.) - die Betelnuß. - Sudhoffs Archiv 50(2): 179-204.

Prentner, A. 2010. Betelnuss und Betel-Pfeffer. In: Bewusstseinsverändernde Pflanzen von A-Z. S. 43-47. - Wien

Schneider, E. 1986. Betel - ein beliebtes Genussmittel Südasiens. Pharmazie in unserer Zeit 15(6): 161-166

Wink, M., van WyK, B. E. \& Wink, C. 2008. Handbuch der giftigen und psychoaktiven Pflanzen. - Stuttgart

\section{Anschrift des Autors}

Herwig Zahorka, JI Bondoga Puri Mas C27, Bogor 16131 Indonesien, E-Mail: zahorka.herwig@gmail.com

\section{Die Zutaten für den Betelbissen}

Die Sirih-Blätter stammen von der Kletterpflanze Piper betle (Piperaceae) auch Betelpfeffer genannt. Sie enthalten ätherische Betelöle und Gerbstoffe, die antiseptisch, adstringend und erfrischend wirken. Sie enthalten keine Alkaloide. Sie müssen frisch sein.

Die Betel- oder Arecanuss ist die Frucht der Betelpalme Areca catechu (Arecaceae). Sie enthält mehrere Alkaloide, wobei das Arecolin den Hauptwirkstoff darstellt, der zusammen mit dem Kalk den Speichelfluss aktiviert und durch Stimulation des zentralen Nervensystems zu einer leichten Euphorie führt. Es macht abhängig, ähnlich wie Nikotin.
Gambir wird aus den Blättern und Zweigen der Kletterpflanze Uncaria gambir (Rubiaceae) hergestellt. Es gibt zwei Verfahren: Die Blätter können abgebrüht, getrocknet, gepresst und zu kleinen Portionen geformt werden, oder Blätter und Zweige werden lange gekocht und der Saft eingedickt, getrocknet und ebenfalls zu Portionen geformt. Die Pflanze enthält Tannine, die antioxidativ und adstringend wirken und Darmparasiten abtöten. Mit Kalk zusammengebracht, entsteht die rote Farbe beim Priemen. Kalk in Form von Kalziumhydroxid (gelöschter Kalk) alkalisiert die Substanzen von Areca und Uncaria, was zu einer besseren Absorption durch die Mundschleimhaut führt. 